\title{
The LDMX Experiment
}

\author{
Jeremiah Mans ${ }^{1, \star}$ for the LDMX collaboration \\ ${ }^{1}$ University of Minnesota, Minneapolis, MN, USA
}

\begin{abstract}
The thermal-relic mechanism for the production of dark-matter in the early universe, combined with the precision measurements of the cosmic microwave background and the lack of observation of dark matter at the LHC, motivates the search for dark matter in the mass range between $1 \mathrm{MeV}$ and $1 \mathrm{GeV}$. We describe the concept for the Light Dark Matter Experiment (LDMX), which could carry out such a search using the missing-momentum technique. The experiment would utilize a unique high-rate, low-current electron beam with an energy between $4 \mathrm{GeV}$ and $10 \mathrm{GeV}$, and would have sensitivity to the thermal-relic hypothesis over a wide range of masses below $1 \mathrm{GeV}$.
\end{abstract}

\section{Introduction}

While the existence of dark matter in the universe is well-established through astrophysical observations including galaxy rotation curves[1], gravitational-lensing studies[2], and measurements of the cosmic microwave background[3], the discovery of the particle which makes up that dark matter has remained stubbornly difficult. Many possible theoretical frameworks have been developed to explain the nature of dark matter and how it was produced in the early universe[4]. Among the simplest possibilities is that cosmological dark matter arose as a thermal relic from the hot early Universe, a concept which only requires small non-gravitational interactions between dark and familiar matter, and is robustly viable over the $\mathrm{MeV}$ to $\mathrm{TeV}$ mass range.

To achieve the dark matter abundance established by cosmic microwave background measurements, the thermal relic process generically requires a weaker coupling between the dark matter and normal matter when the mass of the dark matter particle is lower as number density of relic dark matter particles is proportional to their annihilation cross-section into normal matter. To date, most experimental results have focused on dark matter particle masses in a range from a few $\mathrm{GeV}$ to approximately one $\mathrm{TeV}$, the mass range consistent with the weak coupling scale of the Standard Model. These results include both direct-detection of collisions of dark matter with nuclear targets and searches for direct production of dark matter in colliders. To date, these searches have excluded the thermal relic hypothesis for a wide range of masses [5].

However, these measurements are difficult to extend to lower masses. For colliders, the decrease in coupling predicted by the thermal relic hypothesis reduces the production rate and the low mass hinders the clear identification of the process. For direct-detection experiments, the recoil

\footnotetext{
^e-mail: jmmans@physics.umn.edu
}

of a nucleus due to a sub-GeV mass particle impact is very difficult to measure. However, the sub-GeV mass scale is very natural from analogy with normal matter - indeed most of the electrically-charged fermions have masses in this range. A natural minimal model of dark-matter interactions contains a vector mediator $A^{\prime}$ boson and a neutral dark matter particle $\chi$ which may be either a scalar or fermion [6]. The $A^{\prime}$ will be assumed to mix kinetically with the photon, but there are a wide array of models where the vector coupling arises from a new interaction. For the purposes of dark matter detection, these different models have broadly similar predictions, however, so we will consider the kinetically-mixed $A^{\prime}$ as the example.

Figure 1 shows the limits achieved by colliders, directdetection experiments, and fixed-target experiments, as compared to the targets for either scalar or fermion relic dark matter particles. As can be seen, collider detectors can produce limits over a wide range of masses, but are limited in cross-section sensitivity compared to the thermal-relic target low masses. Direct-detection experiments typically have a low-mass cutoff due to the small amplitude of the recoil compared with the noise. The strongest limits in the low mass region have been established using fixed-target techniques, which allow for very high luminosity to combat the very small predicted crosssection in the thermal relic hypothesis.

The dark matter community has identified this mass region as one of the primary targets for new experiments [8]. Due to the large backgrounds from neutrinos in energetic hadron fixed-target configurations, many of the experiments propose to use electron beams. The production of dark matter particles in the collisions of an electron beam with a dense target is analogous to the usual brehmsstrahlung process in normal matter where an energetic photon is produced in the interaction between a charged particle and the electric field of the nucleus. In the "dark-brehmsstrahlung" process, an $A^{\prime}$ is produced in- 


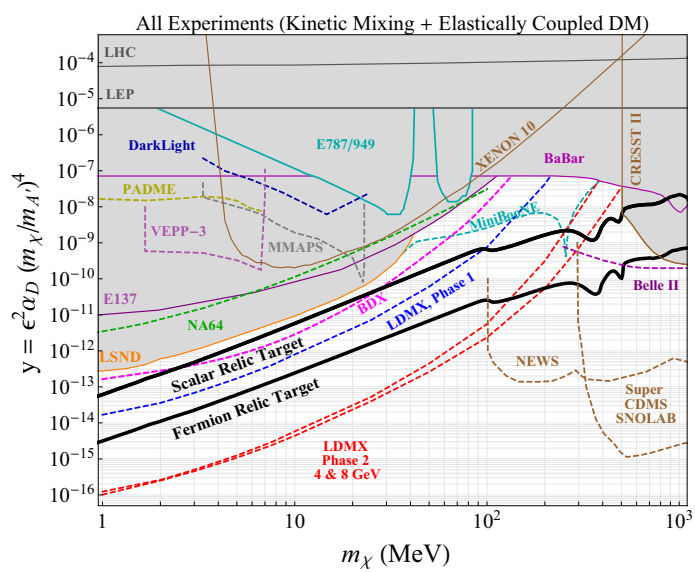

Figure 1. The parameter space for current results (solid areas) and future experimental projections (dashed lines) in the $y$ vs. $m_{\chi}$ plane plotted against the thermal relic targets for scalar and fermion dark matter. The results and projects assume that the mediator couples to either scalar or fermion dark matter which scatters elastically off standard model particles. Results are evaluated for $\frac{m_{\chi}}{m_{A^{\prime}}}=\frac{1}{3}$ and $\alpha_{D}=0.5$ which is a conservative assumption as discussed in [7].

stead, typically through a mixing process with the normal photon. That dark-field mediator then produces a pair of $\chi$ which escape the detector. The kinematics of the "darkbrehmsstrahlung" process are different from the usual process, however. While the normal photon is mass-less, the $A^{\prime}$ boson must have a mass of a similar scale to the $\chi$. As as result, most of the momentum after the collision is typically carried by the $A^{\prime}$ boson and the electron will typically have a moderate transverse momentum after the interaction. This contrasts strongly with normal brehmsstrahlung where the photon energy spans a wide range and is often nearly co-linear with the electron.

The dark-brehmsstrahlung process provides several signatures which can be used for identification of the process[7]. As the $\chi$ particles will carry away most of the energy of the interaction, the experiment can be performed as a missing energy search where the signature is a full-energy incoming beam electron which leaves an anomalously-small energy in the detector. The NA64 collaboration has recently reported results from an initial run of a detector applying this concept using secondary electron beam at CERN [9]. This technique neglects the information which can be gained from the outgoing electron, however. The production of the dark matter will not only cause the electron to lose a great deal of energy, it will also typically result in a significant momentum transverse to the original electron direction. The LDMX experiment proposes to combine the energy-loss of the electron, its transverse momentum after the interaction, and the lack of other activity in the detector to achieve definitive results on the thermal relic hypothesis over a wide range of masses.

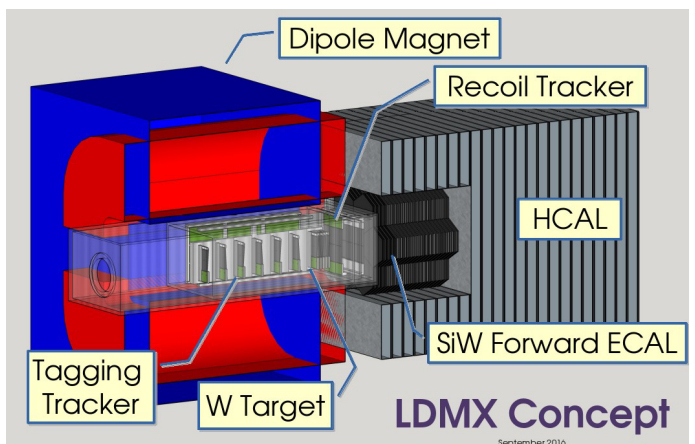

Figure 2. Conceptual layout of the LDMX Experiment.

\section{Experiment Concept}

The LDMX experiment aims to identify events where an incoming beam-energy electron has interacted with a target, with the result of a low-energy, moderate transversemomentum electron and no other observable particles in the final state. This section describes the conceptual design of the detector and initial projections of the detector performance based on simulation studies with simulations based on GEANT4 [10]. The detector design is not final, and adjustments are continuing based on the results of studies, and new performance estimates will be developed. However, the general concept of the detector is established and indicative performance information is available for many of the elements. The projections shown in Fig. 1 are given for two phases of detector operation, phase one with an exposure of $\approx 10^{14}$ electrons-on-target (EOT) and a second phase with $\approx 10^{16}$ EOT.

The design of the detector, shown in Figure 2, is driven by the requirement to fix the kinematics of the interaction precisely and reject an array of background processes which could fake the signal. The experiment consists of a tungsten target in a $1.5 \mathrm{~T}$ dipole magnetic field, preceded by a low-mass tagging tracker and followed by a recoil tracker in the dipole magnet fringe field. The recoil tracker is followed by a high-granularity silicon/tungsten electromagnetic calorimeter and a plastic-scintillator/iron hadron veto calorimeter.

\subsection{Backgrounds}

The primary backgrounds of concern are:

- Beam-related backgrounds - electrons with less than the full-momentum expected in the beam could impact the target, resulting naturally in a low energy observed in the detector. Suppressing this background requires good beam quality and a incoming beam "tagging tracker" to confirm that each incoming electron has the expected momentum.

- Brehmsstrahlung followed by photo-nuclear interaction - the most-common energetic interaction between a beam electron and the target will be a normal 
brehmsstrahlung interaction. For some fraction of these events, the energy transferred to the forward-going photon will be large so that electron energy is low. A calorimeter is needed to identify these photons, but if the photon undergoes a photo-nuclear interaction in the calorimeter, it may leave a relatively-small energy deposit. A sensitive and high-granularity calorimeter is required to identify these photo-nuclear interactions and their showers from the recoil electrons. A hadron veto calorimeter is also needed to contain and identify neutrons and other hadrons produced in the photo-nuclear interactions.

- Neutrino production in the target - in contrast to fixedtarget experiments utilizing hadron beams, neutrino production in electron fixed-target experiments is very low, particularly at energies below $20 \mathrm{GeV}$. For the luminosities required for LDMX, neutrino processes should be unobservable.

\subsection{Beam and Magnet}

To achieve the necessary cross-section sensitivity, the LDMX experiment requires a high-charge, low-current electron beam with an energy in the range of $4-10 \mathrm{GeV}$. The beam would ideally deliver equally-spaced single electrons at a rate of $10^{7}-10^{8}$ electrons per second. Such a beam could be produced using the LCLS II beam-line at SLAC by putting a small number of electrons into the unused accelerator buckets between the primary high-current pulses used for X-ray science. A beamline could be constructed to transport these particles to SLAC's End Station A hall for an experiment. To smooth the radiation load of the electromagnetic calorimeter and allow the spatial separation of multiple electrons in a single accelerator bunch, the beam will be spread to an area of approximately $10 \mathrm{~cm}^{2}$.

The experimental analyzing magnet is a common 18D36 dipole magnet with a $35 \mathrm{~cm}$ vertical gap and operated at a central field of $1.5 \mathrm{~T}$. The magnet is rotated by approximately $100 \mathrm{mrad}$ about the vertical axis with respect to the upstream beamline so that the incoming $4 \mathrm{GeV}$ beam follows the desired trajectory to the target, arriving at normal incidence and centered on the target, which is laterally centered in the vacuum chamber at $40 \mathrm{~cm}$ downstream of the center of the magnet.

\subsection{Tagging Tracker}

The role of the tagging tracker is to ensure that each incoming beam electron has the full beam momentum and arrives at the target at normal incidence. The tagger tracker also measures the impact position of the incoming beam electron, for comparison with tracks in the recoil tracker and clusters in the calorimeters. The design for the tagging tracker is heavily based on the successful design of the HPS tracker [11]. It consists of seven double-sided modules of silicon microstrips arranged at $10 \mathrm{~cm}$ intervals. The modules contain a pair of $4 \mathrm{~cm} \times 10 \mathrm{~cm}$ sensors, with one sensor oriented vertically and the other at $\pm 100 \mathrm{mrad}$ stereo angle, allowing improved pattern recognition and three-dimensional tracking. These standard $p^{+}-$in $-n$ sensors have 30 (60) $\mu \mathrm{m}$ sense (readout) pitch, allowing for excellent spatial resolution.

The readout is based on CMS APV25 ASICs operated in multi-peak mode, which provides reconstruction of hit times with a resolution of approximately 2 ns. At very high occupancies, six-sample readout can be used to distinguish hits that overlap in time down to $50 \mathrm{~ns}$, which limits the readout rate, and therefore the trigger rate, to approximately $50 \mathrm{kHz}$. However, at the low hit occupancies anticipated in LDMX, three-sample readout may suffice, enabling a maximum trigger rate approaching $100 \mathrm{kHz}$. The mechanical and thermal design of the tracker is based on the HPS tracker, but with some more-relaxed requirements due to the lower beam currents and radiation expected in the LDMX experiment.

The tagging tracker design is expected to provide $1 \%$ resolution for $4 \mathrm{GeV}$ incoming electrons. Full simulation of the detector design indicates transverse momentum resolution less than $1.5 \mathrm{MeV}$ along both the bending and non-bending planes, which is small compared with the $4 \mathrm{MeV}$ effect expected from multiple scattering in the target. The impact parameter resolution is expected to be approximately $7 \mu \mathrm{m}(48 \mu \mathrm{m})$ in horizontal (vertical) direction. Finally, simulated $1.2 \mathrm{GeV}$ electrons which are injected such that they can contact all seven layers of the tagging tracker are misidentified as full-energy electrons at a rate of less then $10^{-6}$.

\subsection{Target}

The separation point between the tagging tracker and the recoil tracker is the target. The baseline target design is a $10 \% \mathrm{X}_{\circ}$ tungsten foil which measures $3 \mathrm{~cm}$ by $8 \mathrm{~cm}$. The target is followed by a thin $(2 \mathrm{~mm})$ plastic scintillator plane which is read out directly (without wavelength-shifting) by an array of SiPM phototransducers. This scintillator pad provides information about the energy of fragments emerging from the target and and is used in the trigger as described below. An alternative to the tungsten foil of using an active target based on LYSO is also being considered.

\subsection{Recoil Tracker}

The recoil tracker is designed to identify low-momentum (50 $\mathrm{MeV}$ to $1.2 \mathrm{GeV}$ ) recoil electrons and precisely determine their momentum, direction, and impact position at the target. In addition, it must work together with the calorimeters to correctly distinguish low-momentum signal recoils from scattered beam electrons and multiparticle backgrounds. The key elements of the design are determined by these goals. First, the recoil tracker is placed at the end of the magnet in the beginning of the fringe field to optimize tracking for particles up to two orders of magnitude softer than the beam-energy electrons measured by the tagging tracker. Second, the recoil tracker is short and wide for good acceptance in angle and momentum and to minimize the distance from the target to the 
calorimeters to improve their angular coverage. Finally, the recoil tracker provides 3-d tracking near the target for both direction and impact parameter resolution but emphasizes low mass-density over the longest possible lever-arm further downstream to deliver the best possible momentum resolution. The recoil tracker consists of four stereo layers located immediately downstream of the target and two axial layers at larger intervals in front of the ECAL. The area of the axial layers is significantly larger than that of the stereo layers to maintain angular acceptance. This design delivers transverse-momentum measurements at the $4 \mathrm{GeV}$ resolution limit from multiple-scattering in the target. It maintains $>99 \%$ track reconstruction efficiency for recoil tracks with momentum greater than $1 \mathrm{GeV}$ and falls below $80 \%$ at $\approx 100 \mathrm{MeV}$.

\subsection{Electromagnetic Calorimeter}

In the LDMX design, every beam electron which does not interact in the target can be expected to produce an energetic electromagnetic shower in the calorimeter. As result, the electromagnetic calorimeter must be capable of handling high rates of particle arrival, highly-granular to separate the showers of multiple beam electrons in a single integration interval, and sufficiently radiation-tolerant to absorb the load induced by serving as an active beam dump. To meet these requirements, a high-granularity, Si-W sampling calorimeter will be used for the ECAL of LDMX. The ECAL will draw heavily on the endcap calorimeter upgrade of the CMS experiment for the HL-LHC [12]. In particular, the hexagonal sensors, front-end readout electronics and front-end trigger architecture will be the same. This is facilitated by the fact that the CMS experiment has an instantaneous event rate of $40 \mathrm{MHz}$ while that of LDMX will be $46 \mathrm{MHz}$.

The choice of silicon is important for handling the impact of fairly substantial radiation doses expected in LDMX, particularly in the central sensors of the calorimeter. Simulations of the radiation environment performed with FLUKA [13] indicate that, while electromagnetic interactions are the dominant source of irradiation in the LDMX environment, a substantial hadronic component is also present. For $10^{16}$ EOT, the effective $1 \mathrm{MeV}$ neutron-equivalent fluence at shower max peaks at around $3 \times 10^{15} \mathrm{n} / \mathrm{cm}^{2}$, which is roughly an order of magnitude less than the peak fluence expected for the HL-LHC CMS endcap calorimeter. Such a fluence requires the operation of the silicon at reduced temperature, but appropriate sensor characteristics have been established by the LHC experiments.

The calorimeter design requires 30 layers of $300 \mu \mathrm{m}$ thick silicon sensors with absorber layers of tungsten and copper. Each layer would contain seven hexagonal modules and would be about $51 \mathrm{~cm}$ wide. Because photonuclear backgrounds are most dangerous when the interactions occur as the first or second interaction of an electromagnetic shower, the absorbers will be thinner in the first layers of the calorimeter and thicker after $\approx 3 X_{0}$. This will increase the probability that highly-ionizing fragments from an early photo-nuclear interaction are mea- sured by the silicon rather than being lost in the absorber. The full depth of the electromagnetic calorimeter would be $40 X_{0}$ and approximately $30 \mathrm{~cm}$.

The electromagnetic calorimeter is expected to have a resolution of approximately $17 \% / \sqrt{E} \oplus 1.4 \%$, which will allow good separation of $4 \mathrm{GeV}$ beam particles from recoil electrons, which are expected to have less than 1.2 $\mathrm{GeV}$ of energy. Higher precision is not critical for the experiment, as the recoil tracker will provide excellent momentum measurements for low-momentum recoil electrons. Instead, the calorimeter must have the ability to identify low-activity photo-nuclear events. The excellent MIP-tracking of the calorimeter is important for this effort, as photo-nuclear interactions may result in the production of a small number of charged pions or muons, which can be difficult to identify in some calorimeters.

\subsection{Hadron Veto Calorimeter}

The hadron veto calorimeter is responsible for identifying penetrating hadrons produced from photo-nuclear reactions in the target or the ECAL, particularly energetic neutrons. The occupancy of the calorimeter will be quite low, as relatively few events $(<0.5 \%)$ will produce penetrating hadrons. As a result, the calorimeter can be constructed with limited transverse segmentation to save cost. The active material of the calorimeter is plastic scintillator read out with wavelength-shifter fibers while the absorber material is expected to be iron or steel. The absorber thickness and number of layers are under optimization, however the full depth of the calorimeter is expected to be at least $5 \lambda$, and will need good angular coverage for hadrons initiated either in the target or the electromagnetic calorimeter. The hadron calorimeter will be read out using SiPM-based readout developed for the CMS Phase-1 Hadron Calorimeter Upgrade [14]. Single-neutron inefficiencies of less than $10^{-3}$ are expected for neutrons with kinetic energy above $1 \mathrm{GeV}$.

\subsection{Trigger and DAQ Systems}

The primary trigger for LDMX is based on the electromagnetic calorimeter. The CMS-developed front-end ASIC will produce energy measurements for every $46 \mathrm{MHz}$ accelerator bunch. These energies will be summed over a set of the front layers of the calorimeter, including the shower-max region. The trigger will require that the observed energy be significantly lower than what is expected for a $4 \mathrm{GeV}$ beam electron. Such a trigger can provide a rejection factor of $2 \times 10^{-5}$ with no loss of efficiency for signal. To avoid the trigger firing continuously on empty accelerator buckets, the trigger will also utilize the scintillator pad mounted after the target to identify that a particle passed through the target.

The rejection factor provided by the primary physics trigger is sufficient to allow a total trigger budget of $5 \mathrm{kHz}$, including multiple background-measurement triggers and detector monitoring triggers. The DAQ is designed based on the Reconfigurable Cluster Element (RCE) developed at SLAC [15], and is scaled to be capable of a readout 
bandwidth of $25 \mathrm{kHz}$. The design provides a substantial safety factor in trigger rate and event size, and allows the potential for additional physics triggers to cover other topics.

\section{Conclusion}

The thermal-relic mechanism for the production of darkmatter in the early universe, combined with the precision measurements of the cosmic microwave background and the lack of observation of dark matter at the LHC, motivates the search for dark matter in the mass range between $1 \mathrm{MeV}$ and $1 \mathrm{GeV}$. The Light Dark Matter Experiment (LDMX), which could carry out such a search using the missing-momentum technique. The experiment would utilize a unique high-rate, low-current electron beam with an energy between $4 \mathrm{GeV}$ and $10 \mathrm{GeV}$. It is based on a highlygranular and radiation-tolerant silicon-tungsten calorimeter and a precision tracking system including measurements of both the incoming and recoil electrons. These elements are assisted by a hadronic veto system and a trigger and DAQ system, to complete a design which would have sensitivity to the thermal-relic hypothesis over a wide range of masses below $1 \mathrm{GeV}$. The conceptual design is currently in an advanced state, with design optimization ongoing and performance studies underway using simulated data samples.

\section{References}

[1] V.C. Rubin, N. Thonnard, W.K. Ford, Jr., Astrophys. J. 238, 471 (1980)

[2] D. Clowe, A. Gonzalez, M. Markevitch, Astrophys. J. 604, 596 (2004), astro-ph/0312273

[3] P.A.R. Ade et al. (Planck), Astron. Astrophys. 594, A13 (2016), 1502.01589
[4] H. Baer, K.Y. Choi, J.E. Kim, L. Roszkowski, Phys. Rept. 555, 1 (2015), 1407.0017

[5] T. Marrodán Undagoitia, L. Rauch, J. Phys. G43, 013001 (2016), 1509.08767

[6] M. Pospelov, Phys. Rev. D80, 095002 (2009), 0811.1030

[7] E. Izaguirre, G. Krnjaic, P. Schuster, N. Toro, Phys. Rev. Lett. 115, 251301 (2015), 1505.00011

[8] J. Alexander et al., Dark Sectors 2016 Workshop: Community Report (2016), 1608.08632, http://inspirehep.net/record/1484628/files/ arXiv: 1608.08632.pdf

[9] D. Banerjee et al. (NA64), Phys. Rev. Lett. 118, 011802 (2017), 1610.02988

[10] S. Agostinelli et al. (GEANT4), Nucl. Instrum. Meth. A506, 250 (2003)

[11] P. Hansson Adrian (HPS), The Silicon Vertex Tracker for the Heavy Photon Search Experiment, in Proceedings, 2015 IEEE Nuclear Science Symposium and Medical Imaging Conference (NSS/MIC 2015): San Diego, California, United States (2016)

[12] CMS Collaboration, Tech. Rep. CERN-LHCC2015-010. LHCC-P-008. CMS-TDR-15-02 (2015), https://cds.cern.ch/record/2020886

[13] G. Battistoni, S. Muraro, P.R. Sala, F. Cerutti, A. Ferrari, S. Roesler, A. Fasso, J. Ranft, AIP Conf. Proc. 896, 31 (2007), [,31(2007)]

[14] N.J. Pastika, JINST 11, C03020 (2016)

[15] R. Herbst et al., Design of the SLAC RCE Platform: A general purpose ATCA based data acquisition system, in Proceedings, 21st Symposium on RoomTemperature Semiconductor X-ray and Gamma-ray Detectors (RTSD 2014): Seattle, WA, USA, November 8-15, 2014 (2016), p. 7431254 